\title{
From Smileys to Smileycoins: Using a Cryptocurrency in Education
}

\author{
Gunnar Stefansson, ${ }^{\dagger *}$ Jamie Lentin
}

\begin{abstract}
This paper describes a cryptocurrency to reward students for their studies. The currency bears the apt name Smileycoin or SMLY and is used within the tutor-web online learning platform. In order to make the SMLY attractive to students several approaches have been used, including support from companies whose services can be purchased for SMLY. The paper describes the use of the SMLY as a reward mechanism in a large undergraduate calculus course, including student adoption, student use of SMLY, coinbase use for education in low-income areas, and response to abuse.
\end{abstract}

\section{Introduction}

The tutor-web is a freely accessible online platform for learning. ${ }^{1}$ Although the system contains general educational material (slides, handouts), the main feature is a drilling subcomponent. Here a student logs in to download sets of drill items on given topics using a mobile web interface. ${ }^{2}$ The system forms an adaptive learning environment, ${ }^{3}$ which has been demonstrated to increase student learning, ${ }^{4}$ and has been used as a general vehicle for research on educational technology. ${ }^{5}$

Any educational system eventually needs to have a component to evaluate student performance and the tutor-web is no exception. Such an evaluation will result in a grade, which is also a reward for the student. The purpose of the study reported here is to investigate an alternative model for student rewards.

Within a classroom the typical instructor will use various carrots and sticks, e.g. reward students by acknowledging attempts at problem solving, give high grades for good performance, or draw a "smiley face" on a good solution paper. Although "carrots" tend to be more popular with instructors than "sticks," negative grades for incorrect answers and frowns for cheating are not uncommon methods of giving negative feedback.

Experiments have been conducted on the effects of paying students real money for achieving high grades, ${ }^{6}$ but with conflicting interpretation of the results. ${ }^{7,8}$ Given the current trends in technology, a natural question to ask is whether one can conduct such experiments using electronic currencies such as Bitcoin and what the effect will be of rewarding students using such currencies, ${ }^{9}$ specifically cryptocurrencies as described below.

One famous experiment with electronic currency in education is the MIT Bitcoin experiment, where all undergraduate students were offered \$100 USD worth of Bitcoin, just to

† G. Stefansson (gstefans@gmail.com) is a Professor of Statistics in the Mathematics Department at the University of Iceland.

‡ J. Lentin (lentinj@shuttlethread.com) is the Director of the software consultancy Shuttle Thread Limited, based in the UK. 
investigate the result in terms of its use, adoption, and so forth. ${ }^{10,11}$ The total cost of such an undertaking (over $\$ 300,000$ USD in Bitcoin alone) is prohibitive for most educational research programs. On exactly the same note, research on financial incentives in education relies on having a pool of funds to draw from. Thus the cost of enticing students in a highincome region such as Iceland to work is similarly quite prohibitive and much of this type of research is therefore conducted in low-income regions of the world.

However, it is an easy, cheap, and well-established undertaking to define a new cryptocurrency and in principle this can be used for the same sort of experiments as fiat currency and Bitcoin. Such a currency has therefore been developed for rewards inside the tutor-web. The setup of a new coin was taken on by two undergraduate students over a period of a few months in the summer of 2014, followed by a short period of testing followed by final debugging on a live class in November. The resulting Smileycoin (SMLY) is still in its infancy but initial results are reported here. A new cryptocurrency is by nature a little different from either "smiley faces" or fiat currency, since it is initially of no value, but will gain nonzero value by either being accepted by a merchant or onto a cryptocurrency exchange. Needless to say, there are several issues to consider. For example, in terms of economic incentives, one may ponder over the effects of the (initially) low valuation of the coin as some economic experiments indicate that low rewards may give worse results than no rewards. ${ }^{12}$ Given that cryptocurrencies in general and SMLY in particular are completely new to most students, it is also of interest to see whether the students decide to use this opportunity and whether this use is mostly out of curiosity or only used if the amount to be used is worth their while in monetary terms.

A sister project, Education in a Suitcase, EIAS, is run as a non-profit entity to support education in low-income areas. EIAS is documented in a separate paper but has important links to the SMLY, reported below. ${ }^{13}$

\section{Cryptocurrencies}

Cryptocurrencies use public-private key pairs and the holder of the private key can transfer virtual coins associated with the public key's address to another public address. A software wallet stores addresses and announces transactions stored in a ledger, the blockchain. Blocks are formed by solving cryptographic puzzles and a miner who generates such a block also generates new coins for the system, the coinbase. For Bitcoin, the puzzle algorithm used is based on the SHA-256 cryptographic hash function. As specialized application-specific integrated circuit (ASIC) hardware concentrated mining power, other algorithms emerged, starting with Scrypt, followed by several other algorithms such as Skein.

A new cryptocurrency, the Smileycoin or SMLY, was developed, initially Scrypt-based, as a means to provide incentives to students of the tutor-web. As with any cryptocurrency, the SMLY has a number of parameter settings. This includes the expected frequency of blocks (3 per minute), the premine (target of 50\%) and the coinbase (10,000 SMLY after the premine).

The first block in the SMLY blockchain was mined on 2014-11-13 14:40:29, forming the starting time of the chain. For the next few days a chain was operated while the SMLY were tested within a course at the University of Iceland. Smileycoin was subsequently opened for general mining on November 21, 2014 13:00, ${ }^{14}$ at which time 7025 blocks had been generated. As the coin was not initially open to general mining, these first 7025 blocks constitute the premine. During the premine the block reward was set to 24 million SMLY for the first 1000 blocks, followed by 10,000 during in-class testing and to be used onwards for 
the next 7 years. The SMLY corresponding to the premine were set aside for use inside the tutor-web. The premine was intended to be approximately $50 \%$ of the total but the exact premine, from the 7025 blocks, is $24,060,250,000$ SMLY or $49.6 \%$ of the total computed below.

After the premine phase the block reward was set at 10,000 SMLY, to be halved approximately every 7 years, specifically at every 1,226,400 blocks. Taken literally, this should result in a total of 48,511,868,000 SMLY being generated over a period of approximately 98 years. Of these, $24,451,618,000$ SMLY or $50.4 \%$ will be generated by regular mining.

Given the way halving works, roughly half the remaining total block reward (after the premine) is mined in each 7-year period. Thus miners will generate about 12.2 billion SMLY in block rewards during the first such period.

Difficulty was initially recomputed every 3600 blocks or roughly every 5 days assuming mining hashrate to be in accordance with the difficulty. Vast changes in mining hashrate, further discussed below, led to major changes in difficulty, although they were bounded by a factor of 4. A change in difficulty computation was therefore implemented at block 97050, in August 2015, when difficulty was re-evaluated every 60 blocks instead of the earlier 3600.

SMLY decisions are made in the SMLY bitcoin-talk forum thread. ${ }^{15}$ These include any changes in the mining algorithm, such as the change in difficulty computation.

Table 1. Timeline of SMLY development and changes, indicating testing phase, premine blocks, difficulty adjustment changes, all changes to mining algorithms (from Scrypt-only to adding SHA256, Groestl, Qubit, Skein) and the coinbase split into miners' fee, dividends and donation.

\begin{tabular}{|c|c|c|c|c|c|}
\hline Date & $\underline{\text { Blocks }}$ & $\begin{array}{l}\text { Coinbase, } \\
\text { K SMLY } \\
\end{array}$ & $\begin{array}{c}\text { Coinbase } \\
\text { destination } \\
\end{array}$ & $\begin{array}{l}\text { Difficulty } \\
\text { adjustment } \\
\underline{\text { blocks }}\end{array}$ & $\underline{\text { Event }}$ \\
\hline 13 Nov 2014 & 1 & 24000 & premine & 3600 & First block mined \\
\hline \multirow[t]{2}{*}{ 13-21 Nov 2014} & $2-1000$ & 24000 & premine & 3600 & $\begin{array}{l}\text { Initial premine: } 24 \text { billion } \\
\text { SMLY }\end{array}$ \\
\hline & $1000-7025$ & 10 & premine & 3600 & Testing in single course \\
\hline \multirow[t]{2}{*}{21 Nov 2014} & 7026 & 10 & miners' fee & 3600 & General mining opened \\
\hline & $7026-97050$ & 10 & miners' fee & 3600 & No changes \\
\hline Aug 2015 & 97050 & 10 & miners' fee & 60 & $\begin{array}{l}\text { More frequent difficulty } \\
\text { adjustments }\end{array}$ \\
\hline Jul 2017 & 218000 & 10 & split & 60 & $\begin{array}{l}\text { New wallet, new } \\
\text { algorithms, new coinbase } \\
\text { split }\end{array}$ \\
\hline
\end{tabular}

Block reward-The block reward structure was changed in summer 2017 and made mandatory from block 218,000. Instead of the block reward being used completely for a 
miner's fee, it was split into three parts: the miner's fee, a "dividend" payment, and a donation. Thus the coinbase transaction was changed from normally having a single output to having three outputs. This change followed a migration path from initially being optional through being mandatory if a block is to be accepted into the blockchain. Suggestions on the discussion forum in this context eventually migrated to an implementation where the miner's fee would be reduced by $90 \%$ to 1,000 SMLY and the remaining coinbase of 9,000 be equally split between "dividend" and donation payments.

Although miners are needed to maintain the blockchain, commercial mining is done forprofit only resulting in dumps of coins as the miners reap the mining fee. A "dividend" payment was therefore initiated for SMLY to encourage holding the coin rather than selling it immediately. When a block is mined, addresses containing at least 25 million SMLY are sorted according to when their holding was last modified, i.e. the one with the oldest collection of unused transaction outputs (UTXO) is found. This address receives the entire dividend payment of 4,500 SMLY for this block.

The "dividend" payment is therefore not a regular interest rate or percentage but merely a pre-specified fraction of the block reward. It is of some interest to consider the amounts involved. First, when this was designed, a total of 54 of addresses contained at least 25 million SMLY. However, the total amount officially "available" in March 2017 was approximately 25.8 billion SMLY, ${ }^{16}$ but as seen above, 24.1 of that is a part of the premine, most of which is stored in cold storage. ${ }^{17}$ Thus, some 1.7 billion had been mined and in addition 2.7 billion had been allocated from the premine to students. In addition SMLY can and have been allocated to liquidity provider bots (explained below), wallet developers and for mining rentals, ${ }^{18}$ so the total actually available for general use or purchase was close to 5 billion SMLY. The maximum number of potential 25 million SMLY addresses containing this amount is currently 200. It follows that the short-term number of recipient addresses ("rich" addresses) will be in the range 54-200. As it turned out, from March 2017 until dividend implementation at block 218,000 the number of rich addresses went from 54 to 103 and had increased to 140 by December, 2017.

To evaluate the expected interest rate, consider an intermediate number of 96 holding addresses, to pick a number which rounds nicely. Blocks are intended to be generated once every 3 minutes or 480 every 24 hours. These holding addresses take turns in having the oldest UTXO collection (picking the address with the oldest modification to any UTXO), receiving the interest and thus having the youngest UTXO. With these numbers, each would receive the interest of 4,500 SMLY 5 times daily or 22,500 SMLY/day, giving 8.2 million SMLY per year or a $32 \%$ interest rate. Naturally, doubling the number of addresses, to the maximum current potential, halves the interest rate.

Finally, the donation is a fixed portion of each block, all of which is sent to one address in a rotating list of 10 addresses. The 10 addresses are under the control of the non-profit organization, Education in a Suitcase (EIAS), ${ }^{19}$ which oversees any spending. Formal rules have not been adopted (yet), but discussions in the SMLY forum may well result in a formal approach where votes of the holding addresses can lead to certain projects being paid for from one or more of these addresses (though some addresses will certainly remain permanently for EIAS-specific projects).

The premine-Premine in a cryptocurrency context is a debated topic. In the present case, definitions of acceptable use are laid out on the SMLY home page. ${ }^{20}$ Naturally, rewarding students is the core purpose but SMLY can also be donated to projects likely to increase SMLY use. Development of the allocation process is summarized on the SMLY home page 
(after discussion in the forum). The reward schemes are described below, but the overall aim, after an initial test phase, has been to allocate annually roughly the same amount to students as may be mined within a year. Since reward halving is to take place approximately every seventh year, some 12.2 billion SMLY are to be given as rewards to students in the first 7 years, or about 1.7 billion annually. As will be seen below, considerable tuning of rewards is needed in order to maintain this as an average total.

Alternative methods could certainly have been chosen. For example, instead of a premine the block reward could have been split from the outset, with only a portion becoming the miner's fee and (some of) the remainder going to a tutor-web owned vault.

Liquidity providers-SMLY is listed on cryptocoin exchanges where it receives a market valuation. On these markets the various cryptocoins are treated like stocks on a stock exchange (albeit with little formal regulation).

As with traditional stocks on stock markets, it is important that there be some liquidity, i.e. that there is continuous trade with the coin against one or more other coins. In the absence of liquidity a wide spread can easily form. It is not uncommon to see spreads of orders of magnitude for low-volume coins, severely reducing any options for trading. Some exchanges also have a requirement of minimal liquidity to retain a coin, and this includes the main exchange for SMLY in 2014-2017. Taking all these considerations into account it was deemed necessary to implement a liquidity provider for SMLY.

Liquidity was initially implemented on selected exchanges and markets using automated trading bots. On a given exchange one bot will trade SMLY on several markets, in a loop: $\mathrm{SMLY} \rightarrow \mathrm{DOGE} \rightarrow \mathrm{LTC} \rightarrow \mathrm{SMLY}$, i.e. sell SMLY for DOGE, sell DOGE for LTC and finally sell LTC for SMLY. Another bot running on the same exchange will do this in reverse. In the actual implementation a bot aiming to sell SMLY for DOGE will "look" at the SMLY-DOGE market and first investigate the spread. If the spread is "wide" an offer to sell is made, where an ask price is placed in the middle of the spread. However, if the spread is below a predefined threshold, then a trade occurs. The volume and frequency of these trades is primarily tuned to satisfy the requirement of the exchange in question. As the two bots compete to fill any spread using ask and bid offers followed by sell and buy requests, they maintain low spread and maintain liquidity without an obvious effect on the overall price. The two bots can access more than one exchange and can therefore decide at each stage to buy or sell at the exchange with the best price. This can provide arbitrage between exchanges at no extra cost.

Providing liquidity involves actual trades and offers. Most such trades are simply between the bots so there is little loss involved in each trade. Exchanges do, however, charge a certain percentage of each transaction. Although arbitrage counters this cost, in practice the total costs are always higher than "profits" from arbitrage. The liquidity bots therefore need funding, which is provided from the premine or coins generated from organized mining. ${ }^{21}$

Mining algorithms - The initial use of the Scrypt algorithm proved problematic, since mining pools pointed their pool to and from the SMLY depending on its difficulty and market price. The price of SMLY in 2014-2017 has been strongly linked to BTC, mostly hovering at around 1 Satoshi. Therefore, as Bitcoin prices rose in 2017, so did the dollar price of SMLY. This made SMLY extremely profitable for mining at common Scrypt difficulty in the range 125 and that attracted one or more large mining pools, mining SMLY at extremely high hash rates and leaving once the difficulty increased enough to make mining no longer sustainable. The net effect was that difficulty could have risen by two orders of magnitude once a large 
mining pool left, overwhelming the remaining miners who could take hours or even days to mine a single block, causing the currency to freeze.

For this reason, a new wallet was set up with 5 mining algorithms, SHA256d, Groestl, Scrypt, Skein and Qubit, an approach used earlier by Myriadcoin and Auroracoin. ${ }^{22,23}$ As with other similar coins, the hope is that the multitude of algorithms will reduce the likelihood that the chain will freeze up, though there are certainly mining pools available for all these coins. ${ }^{24}$

\section{Cryptocurrency Within an Educational System}

The Smileycoin was initially developed as a method to test reward mechanisms in the tutor-web educational system and this remains the coin's primary purpose. At the outset this is like any other "funny money," i.e. it is home made and has no intrinsic value, just as Bitcoin was at its inception. To take this idea further, the SMLY logo and name are deliberately designed to indicate that this concept is primarily for fun and not for profit.

Hundreds of cryptocurrencies are listed on cryptocurrency exchanges, ${ }^{25}$ which operate like stock exchanges and one currency can be exchanged for another in different markets. These generate market values, which

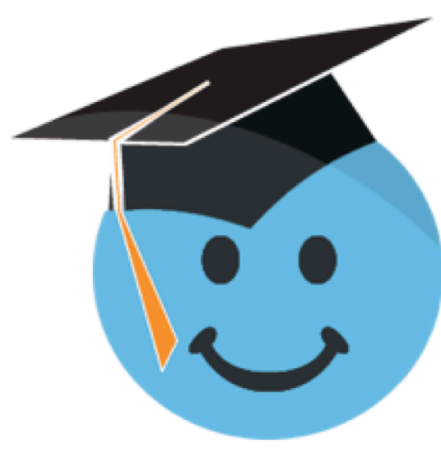

Fig. 1. The SMLY logo are listed on open web pages. ${ }^{26}$ Registering on these exchanges can be done by anyone and is not under the control of the SMLY developers. Thus, SMLY could early on be exchanged for other currencies. For low-valued coins the discrete nature of Bitcoin becomes a problem, as the smallest BTC unit is usually 1 Satoshi $=10^{-8}$ BTC, which became the typical market price of SMLY. It is therefore not a particularly clever idea to buy SMLY for Bitcoin, since that would typically cost 2 Satoshi per SMLY and SMLY could only be sold back for 1 Satoshi, resulting in a $50 \%$ loss. On the other hand SMLY could be sold for Dogecoin, which can be exchanged for Bitcoin, which can be exchanged for US dollars.

With SMLY registered on an exchange it attains the same status as all other cryptocurrencies and changes from "funny money" to something of monetary value. As will be seen below, several other approaches have been used to assign value to the SMLY, but when presented to students it has been a theme throughout that this is primarily for fun.

\section{Your grade: $9 \sim$ Win 10000 SMLY if you ace this lecture, bonus 100000 SMLY for acing whole tutorial}

Fig. 2. The SMLY incentive announcement in the tutor-web.

Rewards within the tutor-web system are tuned by giving students SMLY when certain levels of achievement are reached. Initially these were pre-specified, later optionally chosen from a uniform distribution each time a student started a new topic and finally optionally from a gamma

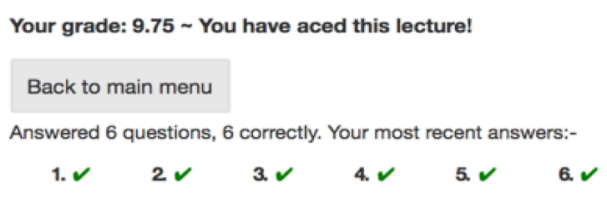

Fig. 3. Announcement of acing a lecture. 
density for a wider range of experimental options. Encouragement is displayed as students answer questions "Win $x$ SMLY if you ace this lecture, bonus $y$ SMLY for acing whole tutorial."

To implement these rewards, the tutor-web system has its own SMLY wallet attached to it, which is topped-up from the pre-mine SMLY, and can automatically distribute SMLY to students using the built-in rules.

There are currently several places in the tutor-web in which students can earn SMLY whilst going about learning activities. The drilling system in tutor-web has been adapted so students get awards for: getting a grade of $50 \%$, getting a top grade in a lecture and getting a top grade in all lectures in a tutorial, with the rewards for each typically going up exponentially.

As with any other part of tutorweb, the rewards are parameterised,

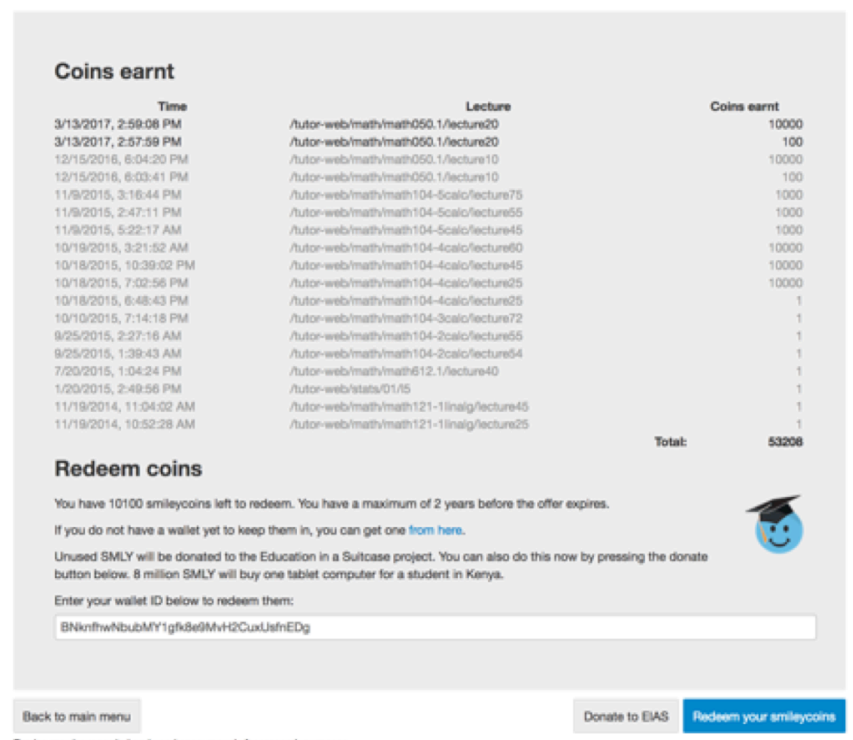

Fig. 4. Student view of SMLY earned in different tutor-web lectures along with buttons for redeeming the SMLY. and can be adapted for each tutorial/lecture. While getting a top grade in a lecture demands good understanding of the material, getting the top grade in the 7-10 lectures that make up a tutorial is correspondingly harder.

Tutor-web maintains a balance of SMLY earned, but this balance is not automatically transferred. The offer is open for 2 years for a student to claim or redeem the balance, thus transferring the balance into their own SMLY wallet, either through tutor-web or directly from within the original tutor-web wallet for Android computers. The amounts involved ( $x$ and $y$ above) are set within each lecture or tutorial, as discussed below, in co-operation between an administrator and an instructor desiring to use the system in conjunction with a real-world classroom.

Drilling is not the only tutor-web activity that has been given SMLY rewards. Wellperforming students are asked to write questions, which will then be given to other students to answer and review, including a grade. If a question is well received by other students (as measured by the peer grade), this also comes with a SMLY reward. Finally there is a studentstudent chat system, where well-performing students can offer help to others, charging them SMLY.

When the SMLY was first introduced, an arrangement was made with a campus coffee shop at the University of Iceland, to permit cups of coffee to be purchased with SMLY. In order to provide further incentives, web pages have been set up, including http://smly.is/ (in Icelandic), where students can buy specific products in the form of electronic coupons directly for SMLY. This approach implies that a hard-working student receives SMLY, which can be used to purchase physical goods. In conjunction with cryptocurrency exchanges this gives a number of ways in which students can use their SMLY. This web page is an ongoing project, where commercial companies are invited to participate by offering coupons for sale. As it 
turns out, companies tend to be quite interested but prefer to donate their coupons rather than receive SMLY and the accompanying accounting issues.

\section{Results}

First responses and data summaries - When introduced to the SMLY, the students' first question asked tends to be skeptical, as in "what can it be used for." Given the answer that one can purchase coffee or various coupons, which give tickets to the cinema, domestic flights or cellular phone airtime, the response of students tends to be incredulity.

The very short experiment at the end of fall semester, November 2014, demonstrated the feasibility of including SMLY in the tutor-web and allocating to students. During those first few weeks there was no way to determine a price to attach to the SMLY nor was there any way to guess the likely number of students who would use the open platform to earn SMLY. To get the experiment started, a cup of coffee was sold for a fixed SMLY price, simply set to correspond to an hour or two of work in the tutor-web. Actual payment for coffee was billed from the project's research account. The use of this option by students was quite minimal, with only a handful of cups of coffee sold.

The same course was next given in fall 2015 and the SMLY was used for the first time for an entire semester. After this trial it became clear that the initial reward settings were too low, with not nearly enough SMLY spent on rewards by the system. The rewards were therefore increased in 2016 with an aim for SMLY spending to achieve the 7-year target of 12.2 billion (1.7 billion/yr). At this time the SMLY was reasonably established on more than one cryptocurrency exchange and the SMLY prices of coffee and coupons were adjusted so that market values for the SMLY and the coupon value roughly matched.

By March 2017 a total of 2.8 billion SMLY had been allocated to students, somewhat below the target, which would be 4 billion for the corresponding 28 months, but of the correct magnitude and can easily be adjusted.

At this stage the entire system is tuned to work like a small economy: The total number of SMLY earned by students are tuned to correspond to halving the "stash" every 7 years. The valuation of the SMLY is done at open exchanges. The valuation of goods sold for SMLY on the smly.is website and coffee shop is set close to the market value of the goods when registered for sale.

The most extensive use of the tutor-web and SMLY has been in a particular course on introductory calculus. ${ }^{27}$ This course has some 450 entrants annually, but in addition all other tutor-web users are also able to earn SMLY, and this currently includes students in high school and graduate school.

The actual rewards given to students can be set within each lecture or across an entire tutorial, in addition to the tutor-web defaults. The only hard limits on the amounts relate to the amount in the system, which imply the goal of spending an average of 1.7 billion/yr. There are quite a number of degrees of freedom in deciding how to set the rewards.

It is considered important for students to move from basic or passing knowledge to excellence and excellence should therefore give much higher rewards than mediocrity. Hence a reward of 1 and 2 million SMLY are typically given for acing a lecture and tutorial, respectively, whereas a much lower reward is given for simply answering a minimal number of questions correctly (possibly ending with a low grade). To "ace" something or get a "top grade" is defined to be "almost perfect", specifically implemented as a grade of 9.75 out of a maximum of 10 . 
A total of 3181 users had earned SMLY from November 2014 through February 2017. These users had earned a total of 2.8 billion SMLY and redeemed 666 million or $24 \%$. It is thus seen that only a small number of students actually redeem their coins, but some students have indeed used their SMLY to purchase movie tickets and a discount at the domestic airline (both through smly.is).

The fact that few students redeem their SMLY may partly be because most students do not get large sums (measured in fiat) as rewards. Thus, although over 500 students have each been awarded 1 million SMLY or more, the value of this is of the order of \$10 USD (using "typical" exchange rates of the time, 100 million SMLY $=1$ BTC $=\$ 1000$ USD). Through March 2017, a total of 34 students had been awarded 25 million SMLY or more, and 25 of those students have redeemed (some of) their SMLY. As expected, students appear more keen to redeem their coins if their coins have more value.

The two pages http://smly.is and http://smly.trade list a few different methods for students to use their SMLY. The smly.is page is somewhat more "official", being the result of formal cooperation between the University of Iceland and commercial companies (in Iceland,), starting with the local airline. The smly.trade page, however, has no official link to the University of Iceland and is more general in where it points SMLY users.

A detailed analysis of where the redeemed SMLY went is not available. However it is known that fewer than 10 movie tickets (coupons) were sold and only one airline coupon was sold. One may speculate on the reasons for this, which may be anywhere from general lack of interest in a technology advertised primarily as fun through greater interest in testing exchanges to not having a reason to use a domestic airline.

Multiple other sites offer different ways to use cryptocurrencies. For example, Novaexhange allows users to use their currency in a small betting setting, including the equivalent of a double-or-nothing game for SMLY. Unfortunately the exchange does not make data available from the use of this option.

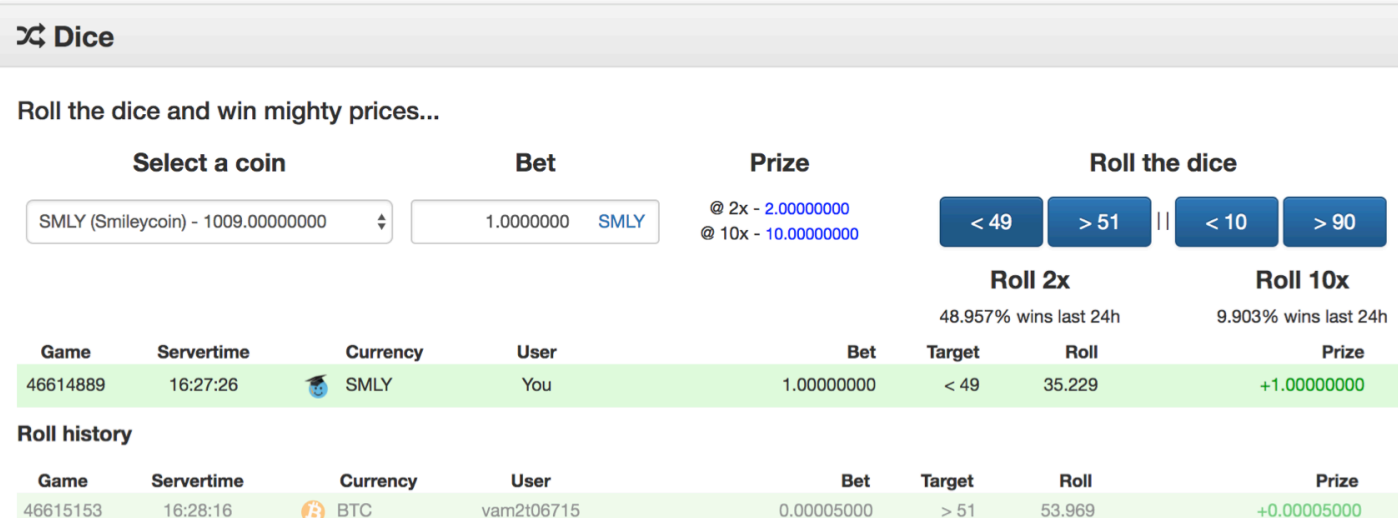

Fig. 5. Dice games at novaexchange.com.

Abuse, faucets and experiments on non-students-Several cases of abuse or attack have been detected. In the first such case a student was found to have set up a handful of email accounts and repeatedly aced one or more introductory lectures, typically lectures with a relatively small set of exercises, and subsequently transferred the SMLY to a wallet. This is an obvious and expected abuse of an open system. In this case the email addresses were 
obviously connected, as were the recipient SMLY addresses. Here, daily monitoring of rewards indicated a deviation in the amount redeemed. This was followed by an investigation into the email addresses corresponding to all wallet recipients of recent extractions of SMLY from the system. As it turned out, all the email addresses were very similar and not only were the SMLY addresses few but they were also linked to each other through transfers. A simple email to the student's addresses was sufficient to stop this first abuse case, but did not stop subsequent attacks.

Although one might consider methods to either auto-detect this sort of abuse, or make it more difficult, it is clear that an attacker could also come up with alternative attack schemes, which would thwart simple detection methods so a different approach is needed. The on-line tutor-web wallet only stores a small portion of the available SMLY and this puts a hard limit on how many SMLY might potentially be stolen from the system. Also, the total allocation and the amount redeemed are both monitored by humans. Since discrepancies are rare, they are simply checked manually. In effect the main guard against abuse is to limit the content of the tutor-web wallet to a magnitude, which will sustain a "run on the bank" by those students who have already earned SMLY. Losing this amount merely corresponds to students redeeming what they own and is a small portion of the available premine.

The second attack was by a more determined student who used multiple email accounts and repeatedly solved the same fairly simple drills, always redeeming to the same SMLY address. A few emails presenting evidence were sufficient to stop this user, even though they initially tried to maintain multiple personalities.

The third attacker was much more elaborate and intent on exploiting the system, using over 200 email addresses and working throughout the entire tutor-web, solving math problems across multiple lectures and tutorials in several languages. As before, the emails were easily linked, as were the (few) SMLY addresses. Email correspondence had no effect on this user's behavior and changes in SMLY reward levels merely moved this person to new - and harder lectures. Since this occurred during summer and outside the school years, all tutor-web parameters could be changed to random settings within very wide ranges. This was done in order to use the abusers to evaluate their responses, i.e. conduct an experiment on the abusers. Simultaneously a reCAPTCHA was installed and a handful of other measures were undertaken as well, including coordinating with the only exchange operating at the time to freeze this particular perpetrator's assets.

These changes, particularly lowering of SMLY rewards, were enough to stop the first wave of abusers but of course some abuse continued. As new and old abusers continued their search for SMLY, data were collected on their behavior in the system. This is done in accordance with the statement which all self-registered students sign, permitting such analyses. These analyses provided the first indications that abusers could be used as a part of a research project on behavior.

A new and intensified wave of abuse started in August 2017. Until mid-October, a total of 317 non-students were seen to use the system, each spending on average 30 seconds on each question and making a total of 210 million SMLY compared to 143 seconds and 101 million SMLY for the 478 regular students (for more detail, see table below).

Here, changing parameter settings resulted in these non-students moving to more profitable topics until they were only using the same lectures as the regular students. At this stage new measures were needed in order to differentiate between "abusers" who merely use the tutor-web to earn SMLY by repeatedly answer the same questions (using different registrations) and students who are there to learn. The solution was to have different parameter 
setting depending on the state of registration. Thus, the system remains open for all, but students who are formally registered in a "tutor-web class" have different parameter settings from those who are not. Class registration is in the hands of vetted instructors.

After this change there is no particular need to worry about abusers. Instead, the selfregistration process is now called a "tutor-web faucet" in accordance with similar "Bitcoin faucets," "Dogecoin faucets," and so forth. It is considered freely available, and the use of multiple email accounts to repeatedly earn a few SMLY is not a problem. The SMLY earnings are simply set differently from the earnings paid out to students in a class. A formal target has not been defined, but initially the total amount of SMLY paid out in the faucet is aimed to be about $10 \%$ of the in-class rewards for the same time periods.

So far there are no known examples of random persons "walking in from the street" and wanting to use the tutor-web seriously as a part of non-school activity. It is nonetheless important for this open-content enterprise to maintain this option. Therefore registration instructions have been modified to explain how an instructor can ask to have an entire class registered and a person can also come off the street and ask to be registered. In both cases the individuals will be vetted by a real person and asked to provide identification. This has not happened in the immediate aftermath of the development of the open tutor-web faucet.

As seen in the following two tables, the outside use of the tutor-web has drastically declined following these changes.

Table 2. Summary statistics August-September 2017 (mostly before converting self-registrations to faucets) for students inside and outside the tutor-web classes.

\begin{tabular}{lrrrrrrrrrr}
\hline domain & MSMLY & timeQ & AveNumQ & redeemed & numStud & totQ & MSMLY/Hr & mins/Q & earn/Q & Q/Stud \\
class & 101 & 143 & 165 & 137 & 478 & 78637 & 42.4 & 0.87 & 1289 & 165 \\
unknown & 5 & 130 & 133 & 6 & 41 & 5453 & 2.4 & 0.98 & 968 & 133 \\
out & 210 & 30 & 146 & 234 & 317 & 46192 & 413.6 & 0.21 & 4550 & 146 \\
\hline
\end{tabular}

Table 3. Summary statistics after converting self-registrations to faucets for students inside and outside the tutor-web classes.

\begin{tabular}{lrrrrrrrrrr}
\hline domain & MSMLY & timeQ & AveNumQ & redeemed & numStud & totQ & MSMLY/Hr & mins/Q & earn/Q & Q/Stud \\
class & 10.365577 & 98.6 & 87 & 0 & 13 & 1136 & 6.3 & 1.13 & 9124.6 & 87 \\
out & 0.000046 & 16.0 & 9 & 14 & 3 & 27 & 0.00 & 1.78 & 1.7 & 9 \\
\hline
\end{tabular}

Simple regression analyses of available data indicate that the number of attempts at answering questions within a lecture are highly significantly different, when compared across users, lectures, SMLY awards, the number of answers used in computing the grade, the shape of the grade weighing function and so forth. The complete analysis of these data falls somewhat outside the scope of this paper, but one interesting conclusion is that results collected from abusers of the system, are reasonably similar to corresponding data from regular students. One particular set of tests is the relation between the amount of SMLY awards and diligence, as measured by the number of attempts within a lecture $(P=0.025$ for abusers and $P<0.001$ for students). 
The primary result from these analyses is that the effect of the reward is on average to increase the number of attempts by approximately 10 attempts on average, for each million SMLY offered (in the range 0-2 million SMLY, for regular students). However, as noted by other authors this is probably not a simple linear function (the data currently available are not sufficient to estimate this relationship as a nonlinear function). ${ }^{7,8}$

Given these results from these experiments with abusers and faucets, tutor-web researchers now have at their disposal many more options and tools than applicable with only regular students. There are considerable limitations on how one can set parameters for students in a regular classroom, since the tutor-web grades typically count towards course grades. There are no such limitations for non-students, however, and it follows that parameters can be set to random values over much wider ranges, exploring portions of the parameter space otherwise inaccessible. Conclusions from the faucet users can then be used for more targeted exploration of improved settings for in-class students. Of course there are some differences in the nature of these user groups and these differences must be kept in mind. For example faucet users in general may simply be interested in math or interested in SMLY (the earlier abusers appeared to be only interested in SMLY), but crucially they are likely to be using the system of their own free will, as opposed to students for whom this is commonly mandatory. Thus the largest student group, the calculus students, consists of non-math majors and most are not particularly keen on math studies.

Obtaining a new faucet-based test-bed for studying the effects of parameters was completely unexpected and was only obtained through the cryptocurrency reward system inside the tutor-web. The faucet development is similarly a unique feature of the system. Being new and of low "revenue" the faucets are only just beginning to be used at the time of this writing (60 users in the first two weeks of October, 2017), but the rewards can easily be tuned upwards, advertised and used more extensively for research.

A totally different type of abuse is the ever-present threat of a $51 \%$ attack. This may have occurred in spring of 2017, when a single large mining pool repeatedly turned to SMLY, mined a large number of blocks and left once difficulty had increased. Whether deliberate or not, large numbers of transactions became orphaned at this time, block reversals were seen and the miners in this pool did not seem to include transactions in their generated blocks. At the same time, deposits were made to exchanges, sold for other coins, the new coin withdrawn and the original SMLY deposit then disappeared, resulting in a pure loss for the exchange. This collective behavior was another reason to modify the wallet to change the coinbase use as well as the mining algorithms. Although likely, the 51\% attack issue was not completely clarified, but these problems have not been seen after the composite mining algorithms were implemented.

Interestingly, the implementation of a pool for SMLY is no longer trivial due to the coinbase change. This was not intended design but a consequence of a desire to use the coinbase differently. Since mining pool software needs to be modified to split the SMLY coinbase, it is much less appealing to add SMLY to an existing pool. In fall of 2017 mining appears to be undertaken only by individuals (and minimal organized CPU mining).

Redeemed coins, diagnostics and price trends-Multiple tools are available to track and diagnose various aspects of cryptocoins. Thus, publicly available blockchain explorers can be used to investigate blocks and transactions and various sites list coin value, averaged across exchanges.

At the end of fall semester, 2016, an interesting downward trend in prices was observed, coinciding with the time when calculus students would be completing their studies, redeeming 
their SMLY and selling them en masse for the first time. This type of behavior in relation to the Bitcoin price seems (qualitatively) different from the behavior of other coins such as Litecoin and Dogecoin.

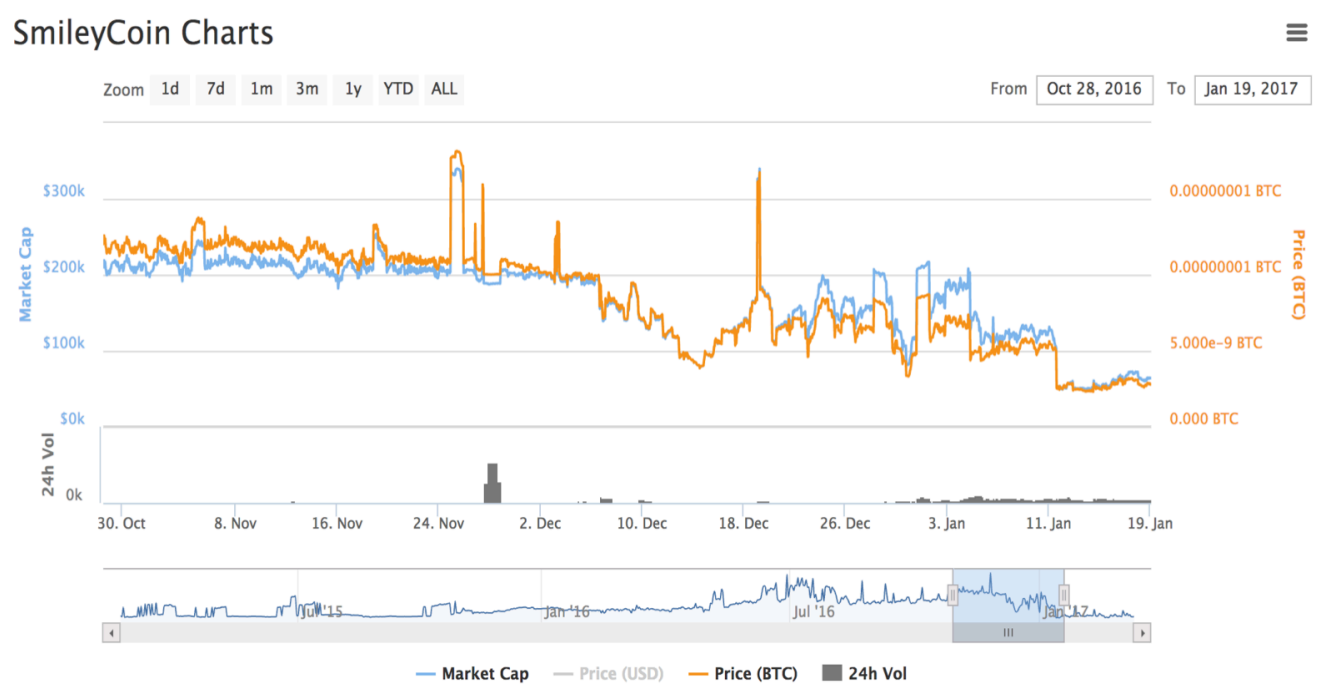

Fig. 6. SMLY price and volume development, around the end of fall semester 2016.

(source: https://coinmarketcap.com/currencies/smileycoin/)

As mentioned above, few students overall redeemed their SMLY in 2016, but 25 out of 34 with over 25 million did so. At the end of fall semester a spike in transactions was observed and at this time these 25 million SMLY were worth about \$125 USD. This was also enough to directly purchase a coupon for an airline ticket discount, movie tickets etc. These may indicate that high rewards are needed to interest students, as seen by other authors. ${ }^{13}$ However, the reason may also be that students who generally master science subjects are more likely to both continue until they receive a high grade and are may also be early adopters of a new technology, but this can not be conclusively seen from these data (other authors have had a separate classifier of early adoption to see behavior of early adopters ${ }^{28}$ ).

With relatively few students wishing to redeem their coins some action was needed to avoid non-redeemed coins accumulating in the tutor-web system. An option was implemented where students can donate their SMLY to Education in a Suitcase, along with a time limit of two years during which students can redeem their SMLY. At the end of the two-year period any non-redeemed SMLY will be automatically donated to EIAS.

In fall, 2016, the opportunity was used to introduce not just the SMLY but also the Education in a Suitcase non-profit operation in the aforementioned calculus course. The response, in the form of applause from the students, was very uncharacteristic for a calculus course. It appeared quite clear that the mere existence of EIAS, along with the implicit student participation in the project through the SMLY, made a positive impression on

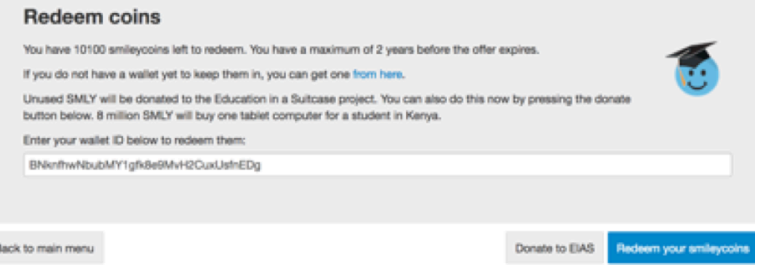

Fig. 7. Option of donating to Education in a Suitcase. many students. As a result, some students decided to donate their earned SMLY during the 
semester, resulting in a total of over 50 million SMLY in donations to the EIAS project in 2016 (\$450 USD value on Jan. 1, 2017).

\section{Discussion}

The traditional reward mechanisms in education have ranged from a pat on the back or a smiley on the student's drawing through exam grades, but as discussed above, experiments have also been conducted using hard currency, e.g. in the form of paying students for grades or for time spent. The approach presented here is to define an electronic coin for rewarding performance within an electronic educational system, and ensure that the coin can subsequently be used outside the system. This comes in addition to the usual perks for correct answers (and negatives for incorrect answers).

The results reported in this paper demonstrate that it is perfectly feasible to bring cryptocurrency into the classroom. Results to date also imply a variable speed of adoption by students, where a few early adopters embrace the coin, others use it for donations but most still ignore the rewards.

If an electronic currency is not perceived to have enough current utility then owners are expected to either ignore or simply sell their coins on a currency exchange, where its price will drop as a result and the same is expected if the owners do not expect future utility either. It is therefore imperative to have several uses available so students have alternatives to dumping the SMLY. The approach to distribute the coinbase partially to large wallet holders, described above, is being tested in part to see whether the resulting interest rate will suffice to at least avoid or counteract coin dumps from students and miners.

One untapped potential of the SMLY is in the Education in a Suitcase project. Since EIAS is used in places like Kenya where a family may only have a single dollar a day for subsistence, a very different perspective opens up. In particular, students who earn \$1 USD in a reasonably short period of time may find new incentives opening up along with a potential for a new level of encouragement from an environment with no tradition of education.

During these first years of developing SMLY rewards, the main goals have been simple system tests and qualitative evaluations of students' responses. A part of the system development has been to set rewards so they very roughly match the target of about 1.7 million SMLY per year. Once achieved, there are several next steps. The primary step is of course to (further) investigate the effect of reward magnitude on learning and learners' behavior. An earlier issue was to tweak the rewards so as to reduce the effects of abuse, but this has now been transformed into studying possibilities of faucets. Ideas from the SMLY forum may lead to reward multipliers for real students who indicate active SMLY participation by registering their wallet addresses within the tutor-web.

Observing non-students (ab)using the system has led to the development of different parameters for self-registered participants, allowing them to earn smaller amounts of SMLY. The existence of faucet users implies that there exist many individuals who will happily answer math questions for SMLY rewards, without being coerced to do so by an instructor or their school. Modifying the tutor-web to work like a faucet for these individuals implies that new experiments can now be set up using only volunteers who give their consent as they register as users in the tutor-web.

Future work includes enhanced services, both economic and otherwise: Services being tested include sending text messages to addresses, through the current blockchain, without requiring a fork. A slight extension to this is to encrypt the messages, after which one can also 
envisage storing students' grades on the blockchain. Once all of this is attained it may become feasible to use student's tablets to sync an entire rural school's set of grades, using any tablet, which gets an Internet connection.

Research questions such as whether the students' response depends on their ability can easily be investigated in this system, as can potential negative effects of low rewards, seen in earlier experiments with economic rewards. ${ }^{8,13}$

Given that dividends are paid only to addresses with a high associated amount, one can also envisage users wanting to group together to reach the minimum amount and to split the rewards. This can, in principle, be implemented through a peer-to-peer lending mechanism, with requests for loans being advertised through text messages within the block chain. Further projects on the drawing board include schemes for tipping or donating using QR codes in public locations.

The tutor-web includes student-submitted questions and their peer-review. These get rewarded, but little research has been conducted on whether they can subsequently be included as a part of the item database in tutor-web. Most of the submitted questions currently appear to be "What is $1+1$ ?" or something similar, but high-quality questions are also submitted. The fact that high SMLY rewards do entice students to work harder for high grades indicates that rewards for good submissions may be a way to receive better submissions and thus make the entire tutor-web more sustainable, requiring much less input from instructors and contentproviders. This is also an interesting avenue of educational research since authoring an item involves a very different learning experience from answering a multiple-choice question. To facilitate this approach, vetted students will be given the final authority to accept submitted items, which other students have deemed of high quality. It remains to be seen what kind of rewards need to be used at each stage of this process, but there are much fewer submissions and reviews, vetted or not, than regular drills so these rewards can be set correspondingly higher.

Finally, an on-going EU-funded tutor-web research project includes development of more options for students to submit material for the tutor-web. One can easily see extensions where students get rewards for submitting general material on arbitrary but predefined topics. This could include material such as examples, completion of proofs of theorems, voting on suggested improvements to existing text and so forth.

\section{Acknowledgement}

The Smileycoin, tutor-web and Education in a Suitcase projects have been funded by several grants and grant agencies including the University of Iceland Research Fund, the Icelandic Research Fund, and EU-funded projects, mostly MareFrame (European Union FP7 grant agreement no. 613571), but also MINOUW (EU H2020 RIA project addressing topic SFS-92014) and FarFish (EU H2020 project).

In addition to SMLY project development and programming by the authors, computer programming has been undertaken by a large number of part-time staff and students, with major individual contributions from Sandra Rós Hrefnu Jónsdóttir, Ívar Örn Ragnarsson, Andrea Björk Björnsdóttir, Ólafur Georg Gylfason, Dagur Tómas Ásgeirsson, Fríða Snædís Jóhannesdóttir, Aníta Kristjánsdóttir, and Hannes Kristinn Árnason.

Considerable feedback on SMLY development has been obtained from a number of colleagues, particularly Anna Helga Jónsdóttir. 
The authors also thank two anonymous reviewers for excellent comments, which have greatly improved the paper.

\section{Author Contributions}

GS and JL both contributed to the manuscript preparation and have overseen teams of programmers implementing the SMLY. JL has programmed the entire current version of the tutor-web, including parameters controlling the SMLY allocation to students and question allocations in the drills. Within the experiments listed, GS has defined parameter values to be used in given classrooms and analyzed the results.

\section{Conflict of Interest}

The authors JL and GS are the primary developers of the Smileycoin. GS has actively traded cryptocurrencies, including SMLY, on various exchanges.

\section{Notes and References}

${ }^{1}$ The tutor-web resource is openly available at https://tutor-web. net and documented in Stefansson, G. "The tutor-web: An educational for system classroom presentation, evaluation and self-study." Computers \& Education 43.4 315-343 (2004) DOI: https://dx.doi.org/10.1016/j.compedu.2003.09.004

${ }^{2}$ Lentin, J., Jonsdottir, A. H., Stern, D., Mokua, V., Stefansson, G. "A mobile web for enhancing statistics and mathematics education." arXiv preprint (2014) https://arxiv.org/pdf/1406.5004.pdf

${ }^{3}$ Jonsdottir, A. H., Jakobsdottir, A., Stefansson, G. "Development and Use of an Adaptive Learning Environment to Research Online Study Behaviour." Educational Technology \& Society 18.1 132-144 ISSN: 1436-4522 (2015) https://www.jstor.org/stable/jeductechsoci.18.1.132

${ }^{4}$ Stefansson, G., Sigurdardottir, A. J. "Web-assisted education: From evaluation to learning." Journal of Instructional Psychology 38.1 47-61, ISSN: 0094-1956 (2011) https : //eric.ed.gov/? id=EJ952150

5 Jonsdottir, A. H. "Development and testing of an open learning environment to enhance statistics and mathematics education." PhD Thesis, University of Iceland. 145 (2015) ISBN 978-9935-9263-0-2 Available at https://skemman.is/handle/1946/20776?locale=en

${ }^{6}$ Levitt, S. D., List, J. A., Neckermann, S., Sadoff, S. "The behavioralist goes to school: Leveraging behavioral economics to improve educational performance." National Bureau of Economic Research. Discussion Paper No. 12-038. 42 (2012) DOI: https://dx.doi.org/10.2139/ssrn. 2084718

${ }^{7}$ Leuven, E., Oosterbeek, H., Klaauw, B. "The effect of financial rewards on students' achievement: Evidence from a randomized experiment." Journal of the European Economic Association 8.6 1243-1265 (2010) DOI: https://dx.doi.org/10.1162/jeea_a_00024

${ }^{8}$ Henry, G. T., Rubenstein, R. "Paying for grades: Impact of merit-based financial aid on educational quality." Journal of Policy Analysis and Management 21.1 93-109 (2002) DOI: https://dx.doi.org/10.1002/pam.1042

9 Nakamoto, S. "Bitcoin: A Peer-to-Peer Electronic Cash System." (2008) https://bitcoin.org/bitcoin.pdf 
${ }^{10}$ Catalini, C., Tucker, C. "Seeding the S-Curve? The Role of Early Adopters in Diffusion" (No. w22596). National Bureau of Economic Research 2016 DOI: https://dx.doi.org/10.2139/ssrn.2822729

${ }^{11}$ Athey, S., Catalini, C., Tucker, C. "Escaping from Government and Corporate Surveillance. Evidence from the MIT Digital Currency Experiment." (2016)

https://www.ftc.gov/system/files/documents/public_comments/2016/10/00071-129190.pdf

12 Gneezy, U.; Rustichini, A. "Pay Enough - Or Don't Pay at All.” CentER Discussion Paper 1998-57

Tilburg: Microeconomics. (1998) DOI: https ://dx.doi.org/10.1162/003355300554917

${ }^{13}$ Stefansson, G., Stern, D. A., Lentin, J., Jonsdottir, A. H. "Evidence-based technology to enhance mathematics education from Iceland to Kenya." Strathmore International Mathematics Conference, SIMC2017, 9 (2017) Abstract DOI: http://dx.doi.org/10.21125/inted.2017.0409

${ }^{14}$ Altcoincalendar is a common method to advertise new coins and this was used for the Smileycoin to try to ensure a transparent method of introduction: https://www.altcoincalendar.info/coins/997-SMLY

${ }^{15}$ Bitcointalk provides a general forum for discussion altcoins as well as Bitcoin, with the SMLY discussion at https://bitcointalk.org/index.php?topic $=845761.0$; all being used to discuss all planned changes to the Smileycoin.

${ }^{16}$ As of December, 2017, the Smileycoin blockchain explorer can be reached at https://chainz.cryptoid.info/smly/

${ }^{17}$ A cold storage or cold wallet is a collection of (private) addresses, which are stored offline.

${ }^{18}$ Technical issues hampered re-implementation of the Android wallet and a wallet from a commercial supplier, Coinomi, became the main Android wallet in summer 2017.

${ }^{19}$ The EIAS project has an official web page at http://educationinasuitcase.com/

${ }^{20}$ The Smileycoin has an official web page at https://tutor-web.info/smileycoin

21 The Science Institute of the University of Iceland (SCUI) is the primary location of research on the effects of Smileycoin use. SCUI therefore maintains some mining to guarantee block generation.

${ }^{22}$ Myriad core developers. Myriadcoin implementation, at https://github.com/myriadcoin/myriadcoin

${ }^{23}$ Auroracoin core developers. Auroracoin implementation, at https://github.com/aurarad/Auroracoin

${ }^{24}$ Such as https : //theblocksfactory.com/

${ }^{25}$ Such as http://c-cex.com and https://novaexchange.com/

${ }^{26}$ On sites such as https://coinmarketcap.com/all/views/all/ where one can also find the Smileycoin

${ }^{27} \mathrm{ST} Æ 105 \mathrm{G} / \mathrm{ST} Æ 108 \mathrm{G}$ at the University of Iceland, from 2014 onwards.

${ }^{28}$ Catalini, C., Tucker, C. "When early adopters don't adopt.” Science 357.6347 135-136 (2017) DOI: https://dx.doi.org/10.1126/science.aal4476

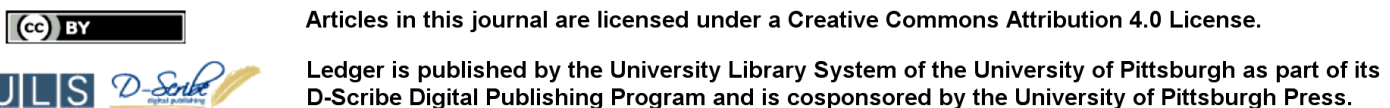
ULLS D-ferle Ledger is published by the University Library System of the University of Pittsburgh as part of its 